\title{
$A b$ initio study of secondary isotope effects on molecular structure
}

\author{
L. S. Bartell and Susan Fitzwater \\ Chemistry Department, The University of Michigan, Ann Arbor, Michigan 48104
}

\author{
Warren J. Hehre* \\ Chemistry Department. The University of California at Irvine, Irvine, California 92664 \\ (Received 17 April 1975)
}

\begin{abstract}
An $a b$ initio calculation of a secondary isotope effect on a bond length has been carried out for the first time. Single determinant molecular orbital computations indicate that the mean $\mathrm{C}-\mathrm{C}$ bond length at $0^{\circ} \mathrm{K}$ is longer in $\mathrm{C}_{2} \mathrm{H}_{6}$ than in $\mathrm{C}_{2} \mathrm{D}_{6}$ by $0.0015 \AA$. A comparison with model calculations supports a steric interpretation in which $\mathrm{H}$ atoms act as if they are bulkier than $\mathrm{D}$ atoms owing to their larger bending amplitudes of vibration.
\end{abstract}

\section{INTRODUCTION}

Primary isotope effects on structure and kinetics are well understood. The increase in length of a bond when one of the atoms is replaced by a lighter isotope can be attributed to the greater amplitude of vibration of the lighter isotope in a Morse-like anharmonic potential well. The greater ease of rupturing a bond containing the lighter isotope is accounted for by its greater zeropoint energy. Secondary isotope effects, in which the length or rate of dissociation of a given bond is altered by isotopic substitution in an adjacent bond, are less well understood. Kinetic isotope effects can arise even if the force fields are perfectly harmonic over the amplitudes of vibrations of the species. ${ }^{2}$ In the case of mean $\left(r_{s}\right)$ bond lengths, however, only anharmonic components of the force field can give rise to isotope effects.

Over a decade ago, secondary isotope effects on structure were predicted ${ }^{2,3}$ in the course of an exploration of possible steric isotope effects in kinetics. Estimates of magnitudes in hydrocarbons were made on the basis of crude guesses of nonbonded potential functions ${ }^{4}$ and the deuterium isotope effect on the $\mathrm{C}$...H nonbonded amplitude of vibration. ${ }^{2}$ Measurements by electron diffraction of the difference between the $\mathrm{C}-\mathrm{C}$ bond length in $\mathrm{C}_{2} \mathrm{H}_{6}$ and $\mathrm{C}_{2} \mathrm{D}_{6}$ were consistent with the steric prediction but were of limited precision. ${ }^{5}$ Microwave studies by Schwendeman and Kelley of $\mathrm{C}-\mathrm{X}$ lengths in $\mathrm{CH}_{3} \mathrm{X}$ and $\mathrm{CD}_{3} \mathrm{X}$ gave comparable results. ${ }^{6}$ In view of the impor tance of such information in spectroscopic determinations of molecular structures if structure parameters of known physical significance are desired, ${ }^{7}$ it seemed worthwhile to initiate an ab initio molecular orbital computation of the relevant cubic force constants in a prototype case. The present work investigates ethane at the single determinant, 4-31G level, ${ }^{8}$ and compares results with those calculated according to a steric model based on geminal C ...H interactions. ${ }^{9}$ The numerical evaluation of the force constants is described elsewher $e^{10}$ with a full tabulation of results.

\section{THEORY}

From a representation of the ethane force field

$$
V=\frac{1}{2} \sum_{i} \sum_{j} F_{i j} S_{i} S_{j}+\sum_{i \leqslant j \leqslant k} \sum_{F_{i j k}} S_{i} S_{j} S_{k}+\cdots
$$

in terms of symmetry coordinates, ${ }^{11} S_{i}$, the effect of molecular vibrations on the mean displacements $\left\langle S_{i}\right\rangle$, can be derived by setting the generalized forces $\left\langle-\partial V / \partial S_{j}\right\rangle$ equal to zero. ${ }^{12}$ The result, through cubic constants, may be written as

$$
\mathbf{F}\langle\mathbf{S}\rangle=\boldsymbol{F}
$$

or

$$
\langle\mathbf{S}\rangle=\mathbf{F}^{-1} \mathfrak{F}
$$

in which the elements of the column matrix $\langle\mathbf{S}\rangle$ are the mean displacements of the totally symmetric symmetry coordinates from the structure of minimum potential energy, $\mathbf{F}$ is the force constant matrix, and the column matrix $F$ with elements

$$
\mathcal{F}_{i}=-3 F_{i i i}\left\langle S_{i}^{2}\right\rangle-2 \sum_{j \neq i} F_{i i j}\left\langle S_{i} S_{j}\right\rangle-\sum_{j \neq i} F_{i j j}\left\langle S_{j}^{2}\right\rangle
$$

may be viewed as representing a vibrational stress due to the mean-square atomic displacements acting in concert with the anharmonic force constants. Equation (2a) is, in a sense, an expression of Hooke's law applied to internal thermal stress. For a particular coordinate, for example, the $\mathrm{C}-\mathrm{C}$ bond stretch $\left(r-r_{\theta}\right)_{\mathrm{CC}}=S_{3}$, the isotope effect, $\Delta\left\langle S_{3}\right\rangle$, can be determined by means of Eq. (3) with the isotopic shifts in the mean-square amplitudes, together with Eq. (2). The result is

$$
\Delta\left\langle\mathrm{S}_{3}\right\rangle=\Delta\left[\mathcal{F}_{3}-F_{13}\left\langle S_{1}\right\rangle-F_{23}\left\langle S_{2}\right\rangle\right] / F_{33}
$$

or

$$
\Delta\left\langle S_{3}\right\rangle=\left(F^{-1}\right)_{31} \Delta F_{1}+\left(F^{-1}\right)_{32} \Delta F_{2}+\left(F^{-1}\right)_{33} \Delta F_{3} .
$$

Equation (4b) is more useful for computation, while (4a) is more convenient in interpreting results. All of the quantities in Eq. (4) can be evaluated from the ab initio calculations, directly or via a perturbed normal coordinate analysis. In numerical computations we have made the following simplifications. First, the mean-square amplitudes of vibration were calculated from the quadratic force field instead of the full anharmonic force field. The validity of this approximation is discussed in Ref. 12. Secondly, the mean-square amplitudes at $0^{\circ} \mathrm{K}$ and $298^{\circ} \mathrm{K}$ were computed from the published experimental $L$ matrices of Duncan ${ }^{11}$ instead of from the theoretical $F$ matrix because the experimental and theoretical force fields are in adequate agreement. Results for the $\Delta\left\langle S_{\boldsymbol{i}}^{2}\right\rangle$ 
TABLE I. Differences in mean-square amplitudes of symmetry coordinates between protiated and deuterated ethane. ${ }^{2}$

\begin{tabular}{|c|c|c|c|c|}
\hline \multirow[b]{2}{*}{ Symmetry } & \multirow[b]{2}{*}{$i, j$} & \multicolumn{2}{|c|}{$10^{3} \times \Delta\left\langle S_{i} S_{j}\right\rangle$} & \\
\hline & & $0^{\circ} \mathrm{K}$ & $298^{\circ} \mathrm{K}$ & \\
\hline \multicolumn{5}{|l|}{$\overline{A_{1 g}}$} \\
\hline $\mathrm{CH}$ & 1,1 & 1.67 & 1.67 & $\AA^{2}$ \\
\hline $\mathrm{CCH}$ & 2,2 & 5.88 & 5.70 & $\mathrm{rad}^{2}$ \\
\hline $\mathrm{CC}$ & 3,3 & 0.06 & 0.05 & $\AA^{2}$ \\
\hline$\cdots$ & 1,2 & -0.28 & -0.30 & $\AA \cdot \mathrm{rad}$ \\
\hline$\cdots$ & 1,3 & 0.03 & 0.03 & $\AA^{2}$ \\
\hline$\cdots$ & 2,3 & -0.16 & -0.08 & $\AA \cdot \mathrm{rad}$ \\
\hline \multicolumn{5}{|l|}{$A_{1 u}$} \\
\hline$\tau$ & 4,4 & 10.0 & 3.8 & $\mathrm{rad}^{2}$ \\
\hline \multicolumn{5}{|l|}{$A_{2 u}$} \\
\hline $\mathrm{CH}$ & 5,5 & 1.73 & 1.73 & $\AA^{2}$ \\
\hline $\mathrm{CCH}$ & 6,6 & 5.84 & 5.70 & $\mathrm{rad}^{2}$ \\
\hline \multicolumn{5}{|l|}{$E_{u}$} \\
\hline $\mathrm{CH}$ & 7,7 & 1.64 & 1.64 & $\AA^{2}$ \\
\hline $\mathrm{HCH}$ & 8,8 & 7.17 & 7.00 & $\mathrm{rad}^{2}$ \\
\hline $\mathrm{CCH}$ & 9,9 & 4.33 & 3.60 & $\mathbf{r a d}^{2}$ \\
\hline \multicolumn{5}{|l|}{$E_{g}$} \\
\hline CH & 10,10 & 1.66 & 1.66 & $\AA^{2}{ }^{\prime}$ \\
\hline $\mathrm{HCH}$ & 11,11 & 7.36 & 7.29 & $\mathrm{rad}^{2}$ \\
\hline $\mathrm{CCH}$ & 12,12 & 3.55 & 3.43 & $\mathbf{r a d}^{2}$ \\
\hline
\end{tabular}

${ }^{2}$ All values except for $\Delta\left\langle S_{4}^{2}\right\rangle$ are computed from the $\mathrm{L}$ matrices in Ref. 11. More figures are listed than are significant.

and $\Delta\left\langle S_{i} S_{j}\right\rangle$ are given in Table I. Except for the meansquare amplitudes in the $\Delta \mathcal{F}_{i}$ elements in Eq. (4), all quantities were taken from the $a b$ initio calculations. The cubic constants are available from no other source.

Values of the $A_{1 g}$ elements of the ab initio F matrix are presented in Table $I$ and isotopic shifts in the thermal stress elements $\mathcal{F}_{i}$ are listed in Table II, as resolved into stretch, bend, and torsion components. Force constants were evaluated at the $a b$ initio equilibrium structure with $r_{\mathrm{CH}}=1.083 \AA, r_{\mathrm{CC}}=1.529 \AA$, and $\angle \mathrm{HCH}=107.7^{\circ}$, which agrees with the estimated (but unknown) true equilibrium structure to within 0.01 A. $5,7,10$

\section{RESULTS}

Deuterium isotope effects on the structure of ethane, derived via Eq. (4) with the quantities listed in Tables I-III, are presented in Tables IV and V. The primary effect on the $\mathrm{C}-\mathrm{H}$ bond length of $0.0044 \AA$ is of the expected magnitude. ${ }^{5,13}$ It is due mainly to Morse anharmonicity. The change in bond angle is small. Of greatest concern in the present work is the secondary isotope effect on the $\mathrm{C}-\mathrm{C}$ bond length. Ab initio cubic constants

TABLE II. $A_{1 \mathrm{~g}}$ elements of the $a b$ initio $4-31 G$ F matrix. a

\begin{tabular}{rrr}
\hline \hline 5.861 & 0.150 & 0.204 \\
& 0.754 & -0.581 \\
& & 4.877 \\
\hline \hline
\end{tabular}

${ }^{2}$ Energy, mdyn $\cdot \AA, S_{1}, S_{3}$ in $\AA ; S_{2}$ in rad.
TABLE III. Components of the vibrational stress, $a b$ initio and geminal steric model.

\begin{tabular}{|c|c|c|c|c|c|c|}
\hline & $\Delta \pi_{1}(\mathrm{r}$ & \multicolumn{4}{|c|}{$\Delta \mathrm{J}_{2}(\mathrm{mdyn} \cdot \AA / \mathrm{rad})$} & $\Delta 5_{3}(\mathrm{mdyn}) \times 10^{3}$ \\
\hline & $0^{\circ} \mathrm{K}$ & $298^{\circ} \mathrm{K}$ & $0^{\circ} \mathrm{K}$ & $298^{\circ} \mathrm{K}$ & $0^{\circ} \mathrm{K}$ & $298^{\circ} \mathrm{K}$ \\
\hline \multicolumn{7}{|c|}{ From $a b$ initio $F_{i j k}$} \\
\hline HCC bend & $\cdots$ & $\ldots 2$ & -4.8 & -4.5 & 9.8 & 9.0 \\
\hline $\mathrm{HCH}$ bend ${ }^{b}$ & $\cdots$ & $\ldots a$ & 5.1 & 5.0 & -0.8 & -0.8 \\
\hline CH stretch & 62.6 & 62.6 & $\cdots$ & $\ldots a$ & -1.5 & -1.5 \\
\hline Torsion & $\cdots$ & $\ldots 2$ & .. & $\cdots^{2}$ & 1.7 & 0.6 \\
\hline Misc. ${ }^{c}$ & 0.1 & 0.1 & -0.1 & -0.1 & 0.9 & 0.8 \\
\hline Total & 62.7 & 62.7 & $0.2^{\mathrm{d}}$ & $0.2^{\mathrm{d}}$ & 10.1 & 8.1 \\
\hline \multicolumn{7}{|c|}{ From steric model $F_{i j k}$} \\
\hline HCC bend & 1.6 & 1.6 & -4.5 & -4.1 & 13.6 & 12.4 \\
\hline $\mathrm{HCH}$ bend & 2.8 & 2.8 & 6.3 & 6.2 & 0 & 0 \\
\hline $\mathrm{CH}$ stretch & $\cdots$ & $\ldots e$ & -0.4 & -0.4 & 5.8 & 5.9 \\
\hline Torsion & 0 & 0 & 0 & 0 & 0 & 0 \\
\hline Misc. $^{c}$ & 0.2 & 0.2 & -0.1 & 0.0 & 0.7 & 0.5 \\
\hline Total & $\cdots$ & $\cdots$ & 1.4 & 1.7 & 20.1 & 18.8 \\
\hline
\end{tabular}

${ }^{\mathrm{a}}$ Neglected.

${ }^{\mathrm{b}}$ Via $S_{8}, S_{11}$.

${ }^{\mathrm{c} C r o s s t e r m s}$ and $\mathrm{CC}$ stretch.
${ }^{\mathrm{d}}$ Neglects $\mathrm{CH}$ stretch.

${ }^{e}$ Not embodied in model. lead to isotopic shifts of $0.0015 \AA$ at $0^{\circ} \mathrm{K}$ and $0.0011 \AA$ at $298^{\circ} \mathrm{K}$ which are similar to empirical results reported previously. ${ }^{5,6}$

Since energies as a function of molecular deformation are the only quantities utilized in the present study, the quantum theoretical results provide no more basis for physical interpretation than would experimental spectra yielding the same potential constants. It is nevertheless worthwhile to consider whether the present calculations support or undermine the basis of the original predictions. $^{2}$ This basis has become the rationale of effects considered to be "steric isotope effects." 14 Fortunately, it is possible to decompose the present results into quite readily interpretable quantities and to compare these with the quantities expected on the basis of a treatment of conventional steric forces. The latter treatment makes use of the nonbonded potential energy functions ${ }^{9}$

$$
V_{\mathrm{HH}}(r)=14.72[\exp (-3.4 r)]-0.3333 r^{-6}
$$

and

$$
V_{\mathrm{CH}}(r)=135.4[\exp (-3.75 r)]-1.076 r^{-6},
$$

(energy, mdyn - $\AA$; distance, $\AA$ ) proposed prior to the present study for an entirely different purpose. ${ }^{9}$ This treatment leads naturally to the values of vibrational stress and strain components identified as steric con-

TABLE IV. Isotope effects on the structure of ethane. Quantities refer to protiated minus deuterated calculated from $a b$ initio cubic constants.

\begin{tabular}{lccl}
\hline \hline Quantity $\times 10^{3}$ & $0^{\circ} \mathrm{K}$ & $298^{\circ} \mathrm{K}$ & Units \\
\hline$\Delta\left\langle S_{1}\right\rangle$ & 10.1 & 10.1 & $\AA$ \\
$\Delta\left\langle S_{2}\right\rangle^{\mathrm{a}}$ & -0.7 & -0.8 & $\mathrm{rad}$ \\
$\Delta\left\langle S_{3}\right\rangle$ & 1.5 & 1.1 & $\AA$ \\
$\Delta\left\langle r_{\mathrm{CH}}\right\rangle$ & 4.4 & 4.4 & $\AA$ \\
$\Delta\left\langle\Delta \psi_{\mathrm{CCH}}\right\rangle^{\mathrm{b}}$ & 1.6 & 0.2 & $\mathrm{deg}$ \\
\hline \hline
\end{tabular}

${ }^{2}$ Neglects effects of $\mathrm{CH}$ stretch and torsion.

${ }^{b} \Delta\langle\Delta \psi\rangle \approx\left(\partial \psi / \partial S_{2}\right)_{0} \Delta\left\langle S_{2}\right\rangle+(1 / 2)\left(\theta^{2} \psi / \theta S_{2}^{2}\right)_{0} \Delta\left\langle S_{2}^{2}\right\rangle$. 
TABLE V. Contributions to secondary isotope effect on $\mathrm{C}-\mathrm{C}$ bond length in ethane, thousandths of an angstrom unit at $0^{\circ} \mathrm{K}$.

\begin{tabular}{lccc}
\hline & \multicolumn{2}{c}{$\begin{array}{c}\text { Component } \\
\text { Effective size }\end{array}$} \\
Vibration & $a b$ initio & Steric & $\begin{array}{l}\text { Effective position } \\
a b \text { initio }\end{array}$ \\
\hline HCC bend & 2.0 & 2.8 & $-0.1^{c}$ \\
CH stretch & -0.3 & 1.2 & -0.4 \\
Torsion & 0.3 & 0 & $\ldots$ \\
Misc. & 0.0 & 0.1 & $\ldots$ \\
\hline \hline
\end{tabular}

a $\left[\left(\Delta \mathcal{F}_{3}\right)_{l} / F_{33}\right]$, or effect of mean square amplitudes of type $l$ on thermal stress $\Delta \mathscr{F}_{3}$.

${ }^{b}\left[-F_{i 3} \Delta\left\langle S_{i}\right\rangle / F_{33}\right]$, or effect of mean isotopic displacement of hydrogen atoms. The steric model, devoid of Morse bond stretching anharmonicity, gives no adequate basis for the effective positions of the hydrogen atoms.

${ }^{c}$ Neglects effect of $\mathrm{CH}$ stretch and torsion on $\Delta F_{2}$.

${ }^{\mathrm{d}}$ Crossterms and $\Delta\left\langle S_{i}^{2}\right\rangle$ for $i=3,8,11$.

tributions in Tables III and V. That is, the necessary cubic coupling constants for Eq. (3) can be evaluated for the steric model by differentiating the potential energy if it is assumed that the entire energy consists of the pairwise repulsions (5) acting between nonbonded interaction centers (with $\mathrm{C}-\mathrm{H}$ bonds foreshortened by 0.117 $\AA)$. For simplicity, in this work only geminal $(1 \ldots 3)$ interactions are considered.

\section{DISCUSSION}

In the original treatment of steric isotope effects on molecular structure it was considered that the nonbonded forces of repulsion between carbon and hydrogen atoms in ethane, averaged over molecular vibrations, could be written as

$$
\begin{aligned}
\left\langle f\left(r_{\mathrm{C} \ldots \mathrm{H}}\right)\right\rangle= & f\left(r_{e}\right)+\left(\partial f / \partial r_{\mathrm{C} \ldots \mathrm{H}}\right)_{e}\left\langle\Delta r_{\mathrm{C} \ldots \mathrm{H}}\right\rangle \\
& \left.+1 / 2\left(\theta^{2} f / \partial r_{\mathrm{C} \ldots \mathrm{H}}^{2}\right)_{e}\left\langle\Delta r_{\mathrm{C} \ldots \mathrm{H}}\right)^{2}\right\rangle+\cdots .
\end{aligned}
$$

The second term on the rhs expresses the attentuation of forces with increasing distance and corresponds to the quantities $F_{i j}\left\langle S_{i}\right\rangle$ in Eq. (2a) and (4a), and to the "effective position" contributions in Table $V$. The last term on the rhs of Eq. (6) expresses the fact that the mean repulsive force, averaged over molecular vibrations, is greater the greater the amplitude of vibration owing to the positive curvature of the force function. This term (or its ab initio representation) is broken down into various physical components in Eq. (3). It is responsible for the vibrational stress in the first place and leads to the entries designated in Table $\mathrm{V}$ as components of the "effective size" of hydrogen atoms.

The fact that the vibrational stress components listed in Table III for $a b$ initio theory and for the geminal steric model are in semiquantitative agreement for the bending modes suggests that the steric model has a substantial element of truth in it. The steric model, however, fails to work for the vibrational stress due to $\mathrm{C}-\mathrm{H}$ stretching, giving an incorrect sign for $\Delta \mathscr{F}_{3}$. That is, the steric cubic constants $F_{i i 3}$, with $i$ a $\mathrm{C}-\mathrm{H}$ stretch, differ in sign from the corresponding $a b$ initio constants. This may be related to a similar observation in the case of $F_{i j}$ constants for hydrides where stretch-bend interactions were found to be correlated with steric forces but where stretch-stretch interactions were dominated by an opposing factor whose origin has been speculated upon elsewhere. ${ }^{10,15}$ In any event, the purely stretching $F_{i i 3}$ are less important than the bend-bend-stretch interactions in the secondary isotope effect.

The zero-point $\mathrm{C}_{2} \mathrm{D}_{6}$ to $\mathrm{C}_{2} \mathrm{H}_{6}$ shift of $\Delta\left\langle r_{\mathrm{CC}}\right\rangle=0.0015 \AA$ based on $a b$ initio cubic constants can be compared with the original crude steric prediction ${ }^{2}$ of $0.003 \AA$, with the experimental electron diffraction value ${ }^{5}( \pm \sigma)$ of $\Delta\left\langle r_{\mathrm{CC}}\right\rangle$ $=0.0016 \pm 0.0007 \AA$, and with the methyl halide values of Schwendeman and $\mathrm{Kelley}^{6}$ of $2 \Delta r_{\mathrm{CC} 1}=0.0016 \AA$ and $2 \Delta r_{\mathrm{CB} r}=0.0020 \AA$. Factors of 2 are included in the latter two values to compensate, approximately, for the fact that there are only half as many hydrogen interactions in the methyl halides as in ethane.

While it is interesting and probably significant to find that the above experimental and theoretical values are in substantial agreement with each other, none of the values are unequivocally reliable. The microwave distances are from $r_{s}$ structures, the precise physical meaning of which is obscure. Sometimes $r_{s}$ lengths are considered to be "close to" equilibrium lengths $r_{e}$, but true equilibrium lengths would show no isotope effect in the BornOppenheimer potential surface. The $a b$ initio cubic constants, while probably close to the true SCF values, may be somewhat in error because of the distortion of the potential surface associated with the fact that SCF wavefunctions lead to incorrect dissociation products. ${ }^{16}$ The calculated steric contributions to the cubic constants seem of reasonable magnitude for the bending modes but not for the $\mathrm{C}-\mathrm{H}$ stretching modes.

\section{CONCLUSION}

The above caveats notwithstanding, it is reasonable to conclude that we have established the magnitude of the secondary deuterium isotope effect upon bond lengths in ethane and related molecules. The new $a b$ initio calculations support the interpretation that a significant component of the effect arises from steric interactions contributed by bending modes. That is, the lighter isotope, vibrating with a larger amplitude, behaves in repulsive interactions as if it is larger than the heavier isotope.

\section{ACKNOWLEDGMENTS}

The $a b$ initio calculations were supported by the Alfred P. Sloan Foundation. Force field calculations and a study of the physical implications were supported by a grant from the National Science Foundation.

\footnotetext{
*Alfred P. Sloan Foundation Fellow.

${ }^{1}$ M. Wolfsberg, Acc. Chem. Res. 5, 225 (1972); E. R. Thornton, Ann. Rev. Phys. Chem. 17, 360 (1966).

${ }^{2}$ L. S. Bartell, Iowa State J. Sci. 36, 137 (1961).

${ }^{3}$ L. S. Bartell, J. Chem. Phys. 36, 3495 (1962).

${ }^{4}$ L. S. Bartell, J. Chem. Phys, 32, $827(1960)$.

${ }^{5}$ L. S. Bartell and H. K. Higginbotham, J. Chem. Phys. 42 , 851 (1965).

${ }^{6}$ R. H. Schwendeman and J. D. Kelley, J. Chem. Phys. 42,
} 
1132 (1965).

${ }^{7}$ K. Kuchitsu, J. Chem. Phys. 49, 4456 (1968).

${ }^{8}$ R. Ditchfield, W. J. Hehre, and J. A. Pople, J. Chem. Phys. 54,724 (1971).

${ }^{9}$ L. S. Bartell and S. Fitzwater (unpublished research).

${ }^{10}$ L. S. Bartell, S. Fitzwater, and W. J. Hehre, "Representations of Molecular Force Fields. I. Ethane: $A b$ Initio and Model, Harmonic and Anharmonic," J. Chem. Phys. (in press).

${ }^{11}$ J. L. Duncan, Spectrochim. Acta 20, 1197 (1964). Note: For the purposes of the present paper, we adopt the curvilinear symmetry coordinates for bends expressed literally in terms of $S_{i}=\sum c_{i j} \Delta \theta_{j}$, linear in the $\Delta \theta_{j}$ (although the dependent $\Delta \theta_{j}$ are not linearly related to the independent $\left.\Delta \theta_{j}\right)$. Our coordinates $S_{i}$, then, are not the more common linear combinations of Cartesian displacement coordinates. Our convention strongly influences the values of the cubic constants $F_{i j k}$ but avoids mixing stretches into the bending coordinates.

${ }^{12}$ L. S. Bartell, J. Chem. Phys. 38, 1827 (1963).

${ }^{13} \mathrm{~K}$. Kuchitsu and L. S. Bartell, J. Chem. Phys, 36, 2470 (1962).

${ }^{14}$ R. E. Carter and L. Melander, Adv. Phys. Org. Chem. 10, 1 (1973).

${ }^{15}$ L. S. Bartell and K. Kuchitsu, J. Chem. Phys。37, 691 (1962).

${ }^{16}$ A. D. McLean, J. Chem. Phys. 40, 243 (1964)。 